\title{
Models of parent-offspring conflict: effect of environmental variance
}

\author{
J. J. Bull
}

Department of Zoology, University of Texas, Austin, TX 78712, U.S.A.

\begin{abstract}
"Parent-offspring conflict" is a theoretical concept proposing that siblings are selected to be slightly more greedy with each other than parents are selected to tolerate. This paper extends a class of previous models of parent-offspring conflict by introducing a new and more realistic dimension to the analysis: the amount of investment an offspring receives is influenced by non-genetic causes (environmental variance) as well as genetic causes. Results of previous models are substantiated for a wide range of conditions, with two interesting results: (1) direct testing of these models of parent-offspring conflict will prove harder than previously supposed, and (2) in some cases the conflict disappears. The models identify several difficulties that confront quantitative, empirical studies of parent-offspring conflict, and they may consequently help anticipate those aspects of parent-offspring conflict that are most amenable to study.
\end{abstract}

\section{INTRODUCTION}

"Parent-offspring conflict" is a term proposed by Trivers (1974) to describe a theoretical result concerning the partitioning of fitness-related resources within a family; the same principle was recognised earlier by Hamilton (1964) in the context of "parental behaviour to minimise sibling competition". In simple terms this theoretical result indicates that an offspring is selected to secure slightly more investment from its parents than parents are selected to give. This theoretical result stems from Hamilton's theory of kin selection (1964): in sexually-reproducing species, siblings are not genetically identical, and selection consequently favours sibs that are mildly selfish over those that share equally. Parents, however, are selected to suppress selfish behaviour among their offspring (assuming that any alleles for preferential treatment of offspring by parents would not be able to discriminate between carrier and non-carrier offspring of those alleles).

Nearly all quantitative studies of parent-offspring conflict have been of models (Trivers, 1974; Parker and MacNair, 1978; 1979; Stamps et al., 1978; Metcalf et al., 1979; MacNair and Parker, 1978; 1979; Hartung, 1980; Charnov, 1982; Feldman and Eshel, 1982; excepting Trivers and Hare, 1976). Even so, it seems that there is yet a rich set of unexplored consequences of parent-offspring conflict, and further models may therefore be use- ful in identifying any unanticipated results. This paper offers some population genetics models of parent-offspring conflict. New results are derived indicating that the empirical measurement of parent-offspring conflict will be more complicated than previously thought, and these results further reveal conditions under which the conflict fails to exist.

\section{A. THE GENERAL MODEL}

In motivating the model, imagine an annual plant that produces its seeds sequentially from stored resources. The number of offspring per plant (family) is limited by this resource, and each family has a total $V$ of this resource. Offspring are produced sequentially during a brief time interval, the $i^{\text {th }}$ offspring obtaining resource $x_{i}$, until all resource is used. Hence, a total of $V / \bar{x}$ offspring are produced, where $\bar{x}$ is the average investment per offspring (it is assumed that $V / \bar{x}$ is sufficiently large that fractional offspring can be neglected). The amount of resource obtained by an offspring, $x_{i}$, in turn influences its probability of surviving to breed according to a function $w(x)$, where $0 \leqq$ $w(x)<1$ for all $x$. All survivors breed in the generation following birth.

The models will investigate the evolution of $x$ when variation in $x$ is inherited according to different alleles at one locus in an outcrossed, 
hermaphroditic population with discrete, nonoverlapping generations. Following most previous treatments of parent-offspring conflict, separate models will be studied for the evolution of $x$ when variation in $x$ is between parents and when variation in $x$ is between offspring. The conclusions reached about parent-offspring conflict will therefore be based on a comparison of the two types of models.

To simplify the algebra, the models will assume that one allele is common and the other rare in the population, and a value of $x\left(x^{*}\right)$ will be sought that satisfies two conditions: (i) when the common allele produces $x^{*}$, no alternative $x$ can successfully invade, and (ii) if an alternative $x$ is common, $x^{*}$ can always invade. These conditions specify an "evolutionarily stable strategy", or ESS (Maynard Smith, 1982). The $w(x)$ function will be assumed fixed in all the models.

In these and previous models, "conflict" takes the form of different values (of $x$ ) selected under parental versus offspring variation. At this qualitative level, parent-offspring conflict has proved robust to various assumptions of a genetic nature (dominant versus recessive control) and of an ecological nature (e.g., sibling competition within versus between broods), although quantitative aspects of the models are certainly influenced by these assumptions. Since the purpose here is to demonstrate that environmental variation (in $x$ ) influences the magnitude of the selected values, this qualitative effect is again not likely to be sensitive to the otherwise restrictive assumptions of the present models.

\section{B. VARIATION BETWEEN PARENTS}

The evolution of investment is straightforward when all heritable variation is between parents according to the amount they invest in offspring. Specifically, suppose that allocation to offspring varies according to the genotype of the maternal parent in each family, independently of paternal genotype. (This assumption embodies the observation that females provide most of the non-genetic resources to offspring in many species.) Let genotypes influence only the average investment, offspring within a family also receiving different investments as a result of "environmental", nongenetic causes. Environmental variance has not been explicitly incorporated in previous models of parent-offspring conflict, and it is the major focus of this study.

The investment received by offspring $i$ in a family whose average (maternal) investment is $y$ is represented as

$$
x_{\mathrm{i}}=y+\delta_{i}
$$

with

$$
E\left[\delta_{i}\right]=0, E\left[\delta_{i}^{2}\right]=\theta^{2},
$$

where $E[\cdot]$ denotes the average or expected value; hence, $E\left[x_{i}\right]=y$. The $\delta_{i}$ term therefore represents the variation around the mean due to environmental (non-heritable) causes.

The average fitness of an offspring from this family is simply the average of $w\left(x_{i}\right)$ taken over all $i$, represented as $E[w(x)]$. Therefore, the average number of surviving offspring from a mother whose average investment is $y$ is

$$
\frac{E[w(y+\delta)] V}{y} .
$$

The ESS value of $y$ is found by maximising (2.B.2) with respect to $y$, here assuming the environmental variance $\left(\theta^{2}\right)$ to be the same across all genotypes (see appendix (i) for a formal derivation of this claim). The ESS value $y^{*}$ therefore satisfies

$$
\left.\frac{\partial E[w(y+\delta)]}{\partial y}\right|_{y=y^{*}}=\frac{E\left[w\left(y^{*}+\delta\right)\right]}{y^{*}}
$$

(assuming that the second derivative of $E[w(x)]$ is continuous and negative at $y^{*}$ ). Result (2.B.3) is equivalent to the result of Smith and Fretwell (1974) and of Parker and MacNair (1978; 1979) except for the consideration of within-mother variance in $x$. Graphically, the solution $y^{*}$ is found by viewing $E[w(y+\delta)]$ versus $y$ in a Cartesian plane, given by the point at which a line through the origin lies tangent to the $E(w(y+\delta)]$ curve (fig. 1).

Previous phenotypic models of parent-offspring conflict of this type have assumed that $\delta=0$, so it is instructive here to consider the effect of environmental variance on the ESS. $w(y+\delta)$ may be expanded around $\delta=0$ by Taylor's series to obtain

$$
w(y+\delta)=w(y)+\delta w(y)+\frac{\delta^{2}}{2}\left[w^{\prime \prime}(y)\right]+\cdots .
$$

Taking expectations,

$$
E[w(y+\delta)]=w(y)+\frac{\theta^{2}}{2}\left[w^{\prime \prime}(y)\right]+\cdots,
$$

(cf. Seber, 1973; Oster and Wilson, 1978; Lacey et al., 1983). As an approximation, this series may be truncated after the second term provided the remainder is sufficiently small. For example, if $\delta$ 


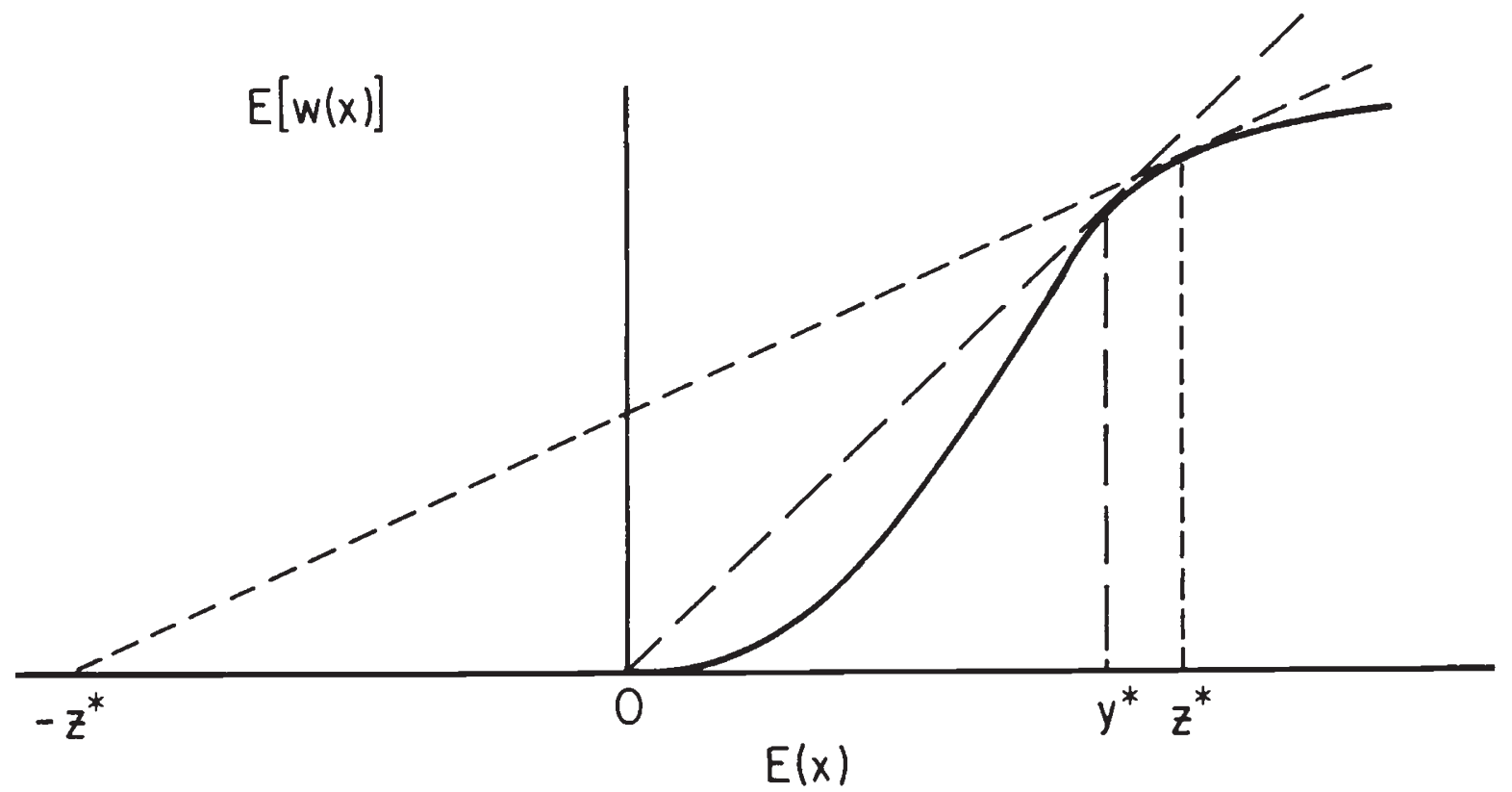

Figure 1 Graphical solutions for parent and offspring ESS allocations to offspring. The parent ESS, $\dot{y}^{*}$, is given by the line through the origin tangent to the curve. The offspring ESS, $z^{*}$, is given by a line tangent to the curve and intersecting the horizontal axis at $-z^{*}$.

is distributed normally with mean zero and variance $\theta^{2}$, odd moments are zero, and even moments are of the form $(2 k) ! \theta^{2 k} /\left(k ! 2^{k}\right)(k=1,2, \ldots)$. The approximation obtained from the first two terms in 2.B.4b may therefore be adjusted arbitrarily close to the true value of $E[w(y+\delta)]$ by choosing an appropriately small $\theta^{2}$, provided that the derivatives of $w(y)$ are appropriately bounded.

From (2.B.4b) and (2.B.3), two effects of $\theta^{2}$ may be observed. First, $\theta^{2}$ may influence the ESS allocation, depending on the form of $w(\cdot)$. Second, increasing $\theta^{2}$ lowers the mean fitness of the family at the ESS. (Since the solution $y^{*}$ is given by a line tangent to the curve from above, $w^{\prime \prime}(y)$ is necessarily negative in this region, and a simple graphical analysis shows that family fitness-given by the slope of the tangent--decreases with $\theta^{2}$ ). Further implications of $\theta^{2}$ will be evident when comparing this result to results when offspring genotypes influence resource allocation, as described next.

\section{C. VARIATION BETWEEN OFFSPRING}

As an alternative to the models in 2.B, investment will now vary according to offspring genotype. The calculations are somewhat less intuitive, however (hence consultation of Appendix (ii) for a more formal derivation may be necessary). In this case, let the level of investment extracted by offspring $i$ be

$$
\begin{gathered}
x_{i}=z+\eta_{i}, \\
\text { with } E\left[\eta_{i}\right]=0, E\left[\eta_{i}^{2}\right]=\theta^{2},
\end{gathered}
$$

where $z$ is the average level of investment taken by offspring of that particular genotype. Here, therefore, $\eta_{i}$ represents the environmental variation investment.

A rare gene $(B)$ in a randomly mating population will occur mostly in families consisting of half heterozygotes, $B b$, and half homozygous $b b$, since homozygous $B B$ individuals and matings of $B b$ with $B b$ are both much rarer than mating of $B b$ with $b b$. Denote the investment parmeters of $B b$ with primes $\left(x^{\prime}\right)$, and those of $b b$ without primes as in (2.C.1). The average fitness of heterozygotes will be $E\left[w\left(x^{\prime}\right)\right]$, and the average number of $B b$ progeny from a typical family with heterozygotes will therefore be

$$
\frac{E\left[w\left(z^{\prime}+n^{\prime}\right)\right]}{\left(z+z^{\prime}\right) / 2},
$$

since the average level of investment in the family is the average of that of $B b$ and $b b$.

The ESS level of investment under offspring variation is found by maximising (2.C.2) with 
respect to $z^{\prime}$, which requires

$$
\left.\frac{\partial E[w(z+\eta)]}{\partial z}\right|_{z=z^{*}}=\frac{E\left[w\left(z^{*}+\eta\right)\right]}{2 z^{*}} .
$$

Equation (2.C.3) differs from the counterpart (2.B.3) only in the factor $\frac{1}{2}$ on the right side. (If all offspring in a brood have different fathers rather than the same father, the factor is ${ }_{4}^{1}$ instead of $\frac{1}{2}$.) Except for the incorporation of error terms, these results are again identical to results from Parker and MacNair (1978, equations 3, 11; MacNair and Parker, 1978: 113 for the result under polyandry). Whereas before the solution $y^{*}$ was found as the tangent to the curve through the origin, the solution here, $z^{*}$, is given by the tangent to the curve from a point to the left of the origin (fig. 1). The effect of environmental variance on the ESS is equalitatively the same for the offspring-control model as for the parental-control model: increasing the variance may influence the ESS and lowers the mean family fitness at the ESS.

Parent-offspring conflict has previously been construed from the existence of different ESS values under the parent- vs. offspring-control models. Here, for example, if the distributions of $\delta$ and $\eta$ are the same, the ESS value under parental control, $y^{*}$ is necessarily less than that under offspring control, $z^{*}$ (provided the second derivative of $E[w(x)]$ is continuous and negative over the regions defined by (2.B.3) and/or (2.C.3)). Thus, as Hamilton (1964) and Trivers (1974) recognised, offspring will evolve to be more selfish than is in the parents' best interests. This comparison of course assumes that the same $w(x)$ function applies under both models - that offspring survival depends on $x$ regardless of how the resource was obtained.

These results reveal a fundamental asymmetry between evolution under parental versus offspring variation, but they do not indicate how systems of investment will evolve when both types of variation are present. Toward this end, the next section presents one type of model for the joint evolution of parental and offspring variation.

\section{INVESTMENT AS A COMBINATION OF PARENTAL AND OFFSPRING VALUES}

Instead of studying separate models for the evolution of $x$, as above, the two types of models may be combined so that investment in each offspring depends on the value of its (maternal) parent and of itself, both of which may evolve. A particularly simple model of this sort describes investment in the offspring as a linear combination of parental and offspring values. For an offspring (i) whose genotypic value is $z$ and mother's value is $y$,

$$
\begin{gathered}
x_{i}=c y+(1-c) z+\gamma_{i} \\
0<c<1 \\
E\left(\gamma_{i}\right)=0 \\
E\left(\gamma_{i}^{2}\right)=\theta^{2},
\end{gathered}
$$

where $c$ is a constant specifying the relative magnitudes of parental versus offspring effect. Here, $y$ evolves according to genetic variation between mothers, and $z$ evolves according to genetic variation between offspring. This model is highly specific and should not be construed as representing any particular biological example; however, the interest here is in a particular type of result, and this result may be general to a wider set of models.

The present model is readily studied with the methods used above, if it is assumed that variation is present in only $y$ or $z$ at any one time, but is sometimes present in $y$ and somtimes present in $z$. (Under weak selection and loose linkage, the simultaneous occurrence of rare genes influencing $y$ and $z$ may also be studied with the above methods, since the two rare alleles will rarely occur in the same individual, of. Feldman and Eshel, 1982). Results from sections 2 and 3 indicated that no value of $E(x)$ can satisfy both the ESS under parental variation and the ESS under offspring variation, given appropriate conditions on the shape of $E[w(x)]$. Therefore, the joint evolution of $y$ and $z$ will be an unending divergence of their values, with the value of $E[x]=c y+(1-c) z$ maintained between or otherwise near the parent and offspring ESS values (see also statements by Parker and Macnair, 1979:1220; and Stamps et al., 1978:384). The divergence continues unabated because each gain made toward one ESS is sooner or later offset by a change toward the other ESS, thus renewing the advantage in evolving toward the first ESS. In fact, the unending divergence of $y$ and $z$ should apply to any $x=f(y, z)$ in which the partial derivatives $\partial f / \partial x$ and $\partial f / \partial y$ do not change sign over all $y$ and $z$ and in which $y$ and $z$ can evolve independently; furthermore, this divergence of values has been observed in a quantitatve genetics model that allowed variation in parent and offspring values simultaneously (Bull, unpublished). 


\section{ENVIRONMENTAL VARIANCE DEPENDENT ON MEAN VALUE}

The models in sections 2 and 3 assumed that the environmental variance, $\theta^{2}$, was independent of the value of $E(x)$. This assumption will now be relaxed. It seems possible on biological grounds, for example, that higher levels of investment may have higher error variances. The above analyses still apply up to the steps (2.B.3) and (2.C.3), at which the ESS's were calculated by differentiation. However, since $E[w(x))]$ is influenced by the environmental variance term, $\theta^{2}$, changes in the mean of $x$ influence $E[w(x))]$ directly and indirectly, by changing $\theta^{2}$. Re-differentiating (2.B.2) and (2.C.2), therefore, leads to the following conditions for EES's:

$$
\begin{aligned}
& \left.\frac{\partial E\left[w\left(y, \theta^{2}\right)\right]}{\partial y}\right|_{y=y^{*}}+\left.\frac{\partial E\left[w\left(y, \theta^{2}\right)\right]}{\partial \theta^{2}} \frac{\partial \theta^{2}}{\partial y}\right|_{y=y^{*}} \\
& =\frac{E\left[w\left(y^{*}, \theta^{2}\right)\right]}{y^{*}}
\end{aligned}
$$

and

$$
\begin{aligned}
& \left.\frac{\partial E\left[w\left(z, \theta^{2}\right)\right]}{\partial z}\right|_{z=z^{*}}+\left.\frac{\partial E\left[w\left(z, \theta^{2}\right)\right]}{\partial \theta^{2}} \cdot \frac{\partial \theta^{2}}{\partial z}\right|_{z=z^{*}} \\
& =\frac{E\left[w\left(z^{*}, \theta^{2}\right)\right]}{2 z^{*}}
\end{aligned}
$$

Criteria for parent and offspring ESS's under the "linear-combination" model of section $3[x=c y+$ $(1-c) z+\gamma]$ can be worked out in similar fashion but the important conclusions can be seen from (4.1) and (4.2).

The term introduced by regarding $\theta^{2}$ as a variable in $E(x)$ admits the possibility of joint solutions to parent and offspring ESS's. Recall that the average family fitness decreases with $\theta^{2}$. If changes in $y$ (or $z$ ) incur a sufficiently large increase in $\theta^{2}$, with consequent reduction in family fitness, selection may maintain a value of $y(z)$ even though it differs from the ESS calculated in (2.B.3) or (2.C.3)-the fitness-effects of changes in $x$ are offset by the fitness-effects of changes in $\theta^{2}$. In the "linear-combination" model of section 3 , the evolutionary divergence of $y$ and $z$ can be halted by increases in $\theta^{2}$, but only if $\partial \theta^{2} / \partial y$ of $\partial \theta^{2} / \partial x$ are sufficient in magnitude.

\section{DISCUSSION}

The models presented in this paper supported previous conclusions that investment in offspring is selected differently according to whether the variation is expressed among parents or among siblings. In agreement with Hamilton (1964) and Trivers (1974), there is a wide range of conditions under which offspring are selected to be slightly selfish with their siblings, whereas parents are selected to eliminate this selfishness (by investing at a level that maximises family fitness). This qualitative result has now proved robust to various models, and consequently, the earlier doubts and misconceptions expressed by some authors about this aspect of parent-offspring conflict seem unwarranted (as reviewed and explained by Parker and MacNair, 1978; Hartung, 1980; Stamps and Metcalf, 1980).

Models of parent-offspring conflict fall into two classes according to whether the gentic variation has been specified in terms of direct fitness increments (costs and benefits), or as here, in terms of phenotypic variation that indirectly translates into fitness increments. (The fitness-increment approach was used in Hamilton, 1964; Trivers, 1974; Stamps et al., 1978; Metcalf et al., 1979; Hartung, 1980; and Feldman and Eshel, 1982; the phenotypic approach was used in Parker and Macnair, 1978; 1979; and Charnov, 1982.) The fitnessincrement approach is undoubtedly the more general, but application of those models to specific biological examples is often difficult without employing the "phenotypic" models as an intermediate, to derive the fitness-increments as consequences of the phenotypic variation.

The models here are similar to those of Parker and MacNair (1978; 1979) except that the present models incorporated environmental variation in the phenotypic parameters. Environmental variances had two important effects on parent-offspring conflict, and these effects comprise the new findings of this paper: (i) the optimal, or ESS, level of investment may be influenced by $\theta^{2}$, so certain types of empirical studies need to consider these variances; and (ii) the nature of the conflict depends qualitatively on properties of the environmental variance.

(i) The first of these points is especially relevant to one empirical method of studying parent-offspring conflict. Having chosen some phenotypic character that may be subject to parent-offspring conflict, it is in principle possible to calculate the parental and offspring ESS levels of investment; the observed level of investment in a population may then be compared to the two ESS values (Trivers and Hare, 1976). The present models illustrated that any calculation of the ESS allocation under parental or offspring variation should 
account for the variance in allocation. (My unpublished quantitative genetics models suggest that the total phenotypic variance may be appropriate rather than just the environmental variance; in the present models the environmental variance was the total phenotypic variance, since the assumption of rare allelic variation forced the genetic variance essentially to zero.) Although the variance may have only a small effect on each of the calculated ESS values, which is determined by the shape of $w(x)$ and the variance in $x$ (from 2.B.3, 2.B. $4 \mathrm{~b}$, and 2.C.3), the degree of "conflict" between parent and offspring is weak enough that the difference between parent and offspring ESS's may itself often be so small that the variance-effect cannot be ignored.

The possible similarity of parent and offspring ESS values in fact seriously challenges the testing of these types of models: after measuring $w(x)$, the variance in $x$, and the relationship between $x$ and brood size, the confidence intervals for parent and offspring ESS's may overlap almost totally, precluding any empirical discrimination of them. Trivers and Hare (1976) identified one problem in which these complications were minimal: workerqueen conflict over the sex ratio in ants. In their case, the fitness function could be derived from the frequency-dependent properties inherent in sexual reproduction and haplo-diploidy, and empirical estimates of fitness were unnecessary (variances in sex ratio were ignored, however). Unfortunately, most phenotypes subject to parentoffspring conflict are likely to require empirical determinations of fitness, thus encountering the possibly insurmountable complications identified here.

(ii) Section 4 described the result that, if changing the mean levels of investment $(y, z)$ also changed their environmental variances, joint ESS's were possible. Mathematically, this result is similar to results in which an outright "cost" was assigned to particular behaviours (Stamps et al., 1978; Parker and MacNair, 1979). Although this variance-effect is potentially important to parent-offspring conflict, some theoretical and empirical questions must be answered before it can be rigorously evaluated. Results (4.1) and (4.2), as well as their analogues for discrete phenotypic variation (unpublished), suggested that rare alleles are selected according to the mean and variance of their effects on the phenotype; a low variance is always advantageous over a higher variance. If true, the possibility of a joint parent/offspring ESS seems remote, since no joint equilibrium satisfying both (4.1) and (4.2) is stable to invasion by a mutation with a sufficiently low environmental variance (and an appropriate mean effect). The theoretical side of this problem is better addressed with models of polygenic characters, rather than with the approach used here. Yet, even with an answer to this theoretical question, the nature of environmental variances in quantitative characters is too poorly understood to anticipate its importance in the evolution of joint ESS's under parentoffspring conflict (Lerner, 1954; Falconer, 1981).

The present analysis, while providing some new results on parent-offspring conflict, highlights the difficulty in obtaining empirical measurements that could be used in direct tests of these and similar models. Even so, models such as these serve at least three useful functions: (i) they identify qualitative results that may be readily observable (e.g., divergence of parent and offspring values), (ii) they define complications in measuring the parameters relevant to parent-offspring conflict and thereby help avoid certain pitfalls, and (iii) as a consequence of (ii), they help anticipate those aspects of parent-offspring conflict that are amenable for study.

\section{APPENDIX: FORMAL DERIVATION OF SELECTION ON RARE GENES INFLUENCING INVESTMENT IN OFFSPRING}

\section{(i) Variation between Parents}

In this first model the maternal genotype will influence the average investment per offspring. Two alleles will be considered, $a$ and $A$, with the former common and the latter rare. Evolution of $A$ may therefore be studied by considering only two genotypes, $a a$ and $A a$, since $\boldsymbol{A A}$ is so rare in comparison to $A a$ that its fitness has a negligible effect on selection of $A$.

The assumptions in sections 2 .A and 2.B may be formalised as:

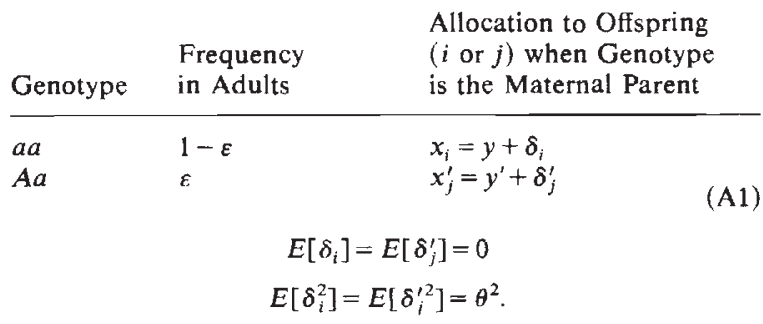

$E[\cdot]$ denotes the expected or average value.

The "linearised" analysis of evolution in this system leads to the following abbreviated compila- 
tion, assuming $\varepsilon \ll 1$; monogamous hermaphrodites are assumed:

\begin{tabular}{|c|c|c|c|c|c|}
\hline \multirow{2}{*}{$\begin{array}{l}\text { Linearised } \\
\text { Frequency } \\
\text { of Mating }\end{array}$} & \multicolumn{2}{|c|}{$\begin{array}{l}\text { Parental } \\
\text { Genotypes }\end{array}$} & \multicolumn{3}{|c|}{ Offspring } \\
\hline & $\sigma$ & $q$ & No. & Survival & Genotype \\
\hline $1-2 \varepsilon$ & $a a$ & $a a$ & $\frac{V}{E[x]}$ & $E[w(x)]$ & $a a$ \\
\hline$\varepsilon$ & $A a$ & $a a$ & $\frac{V}{E[x]}$ & $E[w(x)]$ & $A a / 2+a a / 2$ \\
\hline$\varepsilon$ & $a a$ & $A a$ & $\frac{V}{E\left[x^{\prime}\right]}$ & $E\left[w\left(x^{\prime}\right)\right]$ & $A a / 2+a a / 2$ \\
\hline
\end{tabular}

Here, $E[x]=y, E\left[x^{\prime}\right]=y^{\prime}$, and an approximation for $E[x(\cdot)]$ was derived in (2.B.4).

Ignoring all "second-order" terms, an approximate formula for the change in frequency of $A$ is given by

$$
\varepsilon^{\prime} \simeq \frac{\varepsilon}{2}\left\{1+\frac{E\left[w\left(x^{\prime}\right)\right] / y^{\prime}}{E[w(x)] / y}\right\}
$$

Therefore, $A$ is favored only if

$$
\frac{E\left[w\left(x^{\prime}\right)\right]}{y^{\prime}}>\frac{E[w(x)]}{y},
$$

that is, if the $A a$ mother produces more surviving offspring on average than the $a a$ mother. ((A4) also applies to a model with separate sexes and to the evolution of $A$ at arbitrary frequency when $A$ has a small, additive effect on the average investment.) Thus, if $E[w(x)] / y$ is maximised with respect to $y$ (where $x=y+\delta)$, no alternative value of $y^{\prime} \neq y^{*}$ can invade, hence (2.B.3).

\section{(ii) Variation betweèn Offspring}

This second model differs from the first in that levels of investment are determined by offspring genotype. Inheritance of variation is influenced by a locus with alleles $b$ and $B$, with the latter rare. As in Section A(i) above, the following tabulations

\begin{tabular}{|c|c|c|}
\hline Genotype & $\begin{array}{l}\text { Frequency } \\
\text { in Adults }\end{array}$ & $\begin{array}{l}\text { Allocation to self } \\
\text { (as offspring) }\end{array}$ \\
\hline $\begin{array}{l}b b \\
B b\end{array}$ & $\begin{array}{l}1-\varepsilon \\
\varepsilon\end{array}$ & $\begin{array}{l}x_{i}=z+\eta_{i} \\
x_{j}^{\prime}=z^{\prime}+\eta_{j}^{\prime}\end{array}$ \\
\hline & \multicolumn{2}{|c|}{$E\left[\eta_{i}\right]=E\left[\eta_{j}^{\prime}\right]=0$} \\
\hline & \multicolumn{2}{|c|}{$E\left[\eta_{i}^{2}\right]=E\left[\eta_{i}^{\prime 2}\right]=\theta^{2}$} \\
\hline
\end{tabular}
apply.
From this, for $\varepsilon \ll 1$ and again assuming

\begin{tabular}{|c|c|c|c|c|c|}
\hline \multirow{2}{*}{$\begin{array}{l}\text { Linearised } \\
\text { Frequency }\end{array}$} & \multirow{2}{*}{\multicolumn{2}{|c|}{ Parents }} & \multicolumn{3}{|c|}{ Offspring } \\
\hline & & & No. & Survival & Genotype \\
\hline \multirow{2}{*}{$1-2 \varepsilon$} & \multirow{2}{*}{$b b$} & \multirow{2}{*}{$b b$} & $V$ & & \multirow{2}{*}{$E[w(x)] b b$} \\
\hline & & & $E[x]$ & & \\
\hline \multirow{3}{*}{$2 \varepsilon$} & \multirow{2}{*}{$B b$} & \multirow{2}{*}{$b b$} & & $V$ & $E\left[w\left(x^{\prime}\right)\right] B b$ \\
\hline & & & $\{E[x]$ & $\left.+E\left[x^{\prime}\right]\right\}$ & $E[w(x)] b b$ \\
\hline & & & & 2 & \\
\hline
\end{tabular}
monogamy,

The $B b$ genotype is favored only if

$$
\frac{E\left[w\left(z^{\prime}+\eta^{\prime}\right)\right]}{\left(z+z^{\prime}\right) / 2}>\frac{E[W(z+\eta)]}{z} .
$$

The ESS value of $z$ is therefore by maximising the left side of (A7) over $z$ at the point that $z^{\prime}=z$, justifying (2.C.3).

\section{REFERENCES}

CHARNOV, E. L. 1982. Parent-offspring conflict over reproductive effort. Am. Nat., 119, 736-737.

FALCONER, E. S. 1981. Introduction to Quantitative Genetics. 2nd ed. Longman Group Ltd, Essex, U.K.

FELDMAN, M. W. AND ESHEL, I. 1982. On the theory of parentoffspring conflict: a two-locus genetic model. Am. Nat., $119,285-292$.

HAMILTON, w. D. 1964. The genetical evolution of social behavior. J. Theor. Biol. 7, 1-52.

HARTUNG, J. 1980. Parent-offspring conflict-a retraction. $J$. Theor. Biol., 87, 815-817.

LACEY, E. P., REAL, L., ANTONOVICS, J. AND HECKEL, D. G. 1983. Variance models in the study of life histories. Am. Nat., 122, 114-131.

LERNER, I. M. 1954. Genetic Homeostasis. John Wiley \& Sons, Inc., New York.

MACNAIR, M. AND PARKER, G. A. 1978. Models of parentoffspring conflict. II. Promiscuity. Anim. Behav., 26, 111122.

MACNAIR, M. AND PARKER, G. A. 1979. Models of parentoffspring conflict. III. Intrabrood conflict. Anim. Behav. 27, 1202-1209.

MAYNARD SMITH, J. 1982. Evolution and the Theory of Games. Cambridge University Press, Cambridge, U.K.

METCALF, R. A., STAMPS, J. A. AND KRISHNAN, V. V. 1979. Parent-offspring conflict which is not limited by degree of kinship. J. Theor. Biol. 76, 99-107.

OSTER, G. F. AND WILSON, E. O. 1978. Caste and Ecology in the Social Insects. Princeton University Press, Princeton, N. J, USA.

PARKER, G. A. AND MACNAIR, M. 1978. Models of parentoffspring conflict. I. Monogamy. Anim. Behav. 26, 97-110.

PARKER, G. A., AND MACNAIR, M. 1979. Models of parentoffspring conflict. IV. Suppression: evolutionary retaliation by the parent. Anim. Behav. 27, 1210-1235.

SEBER, G. A. F. 1973. The Estimation of Animal Abundance and Related Parameters. Griffin, London.

SMITH, C. C. AND FRETWELL, S. D. 1974. The optimal balance between size and number of offspring. Am. Nat. 108, 499506. 
STAMPS, J. A. ANI) METCAil.F, R. A. 1980. Parent-offspring conflict. In Sociobiology: Beyond Nature/Nuture?, eds. G. W. Barlow and J. Silverberg, pp. 589-618. Westview Press Inc., Boulder, CO, USA.

STAMPS, J. A. METCALF, R. A., AND KRISHNAN, V. V. 1978. A genetic analysis of parent-offspring conflict. Behav. Ecol. Sociobiol. 3, 369-392.
TRIVERS, R. L. 1974. Parent-offspring conflict. Amer. Zool., 14, 249-264.

TRIVERS, R. L. AND HARE, H. 1976. Haplodiploidy and the evolution of the social insects. Science 191, 249-263. 